\title{
Error Analysis on General Grids for Finite Difference Discretizations of Sturm-Liouville Problems $^{1}$
}

G.S. LORENZZETTI, J.P. ZINGANO, P.R. ZINGANO², Departamento de Matemática Pura e Aplicada, UFRGS, 91509-900 Porto Alegre, RS, Brazil.

\begin{abstract}
We introduce a simple method to obtain very accurate pointwise estimates for both solution and gradient errors of finite difference discretizations on arbitrary grids of one-dimensional Sturm-Liouville problems. Application is given to the detailed analysis of an inconsistent, 2nd-order convergent scheme.
\end{abstract}

Keywords. Sturm-Liouville problems, finite difference methods, error analysis.

\section{Introduction}

In this work we discuss a novel approach for the investigation of error bounds of finite difference approximations on arbitrary grids to smooth solutions of regular Sturm-Liouville problems with separated boundary conditions,

$$
\begin{gathered}
-\frac{d}{d x}\left(\mathrm{~K}(x) \frac{d \mathrm{u}}{d x}\right)+\mathrm{q}(x) \mathrm{u}(x)=\mathrm{f}(x), \quad a<x<b, \\
\alpha_{0} \mathrm{u}(a)-\alpha_{1} \mathrm{~K}(a) \mathrm{u}^{\prime}(a)=\Gamma_{a}, \quad \beta_{0} \mathrm{u}(b)+\beta_{1} \mathrm{~K}(b) \mathrm{u}^{\prime}(b)=\Gamma_{b},
\end{gathered}
$$

where $\alpha_{0}, \alpha_{1}, \beta_{0}, \beta_{1}, \Gamma_{a}, \Gamma_{b}$ are given constants satisfying

$$
\alpha_{0}, \alpha_{1}, \beta_{0}, \beta_{1} \geq 0, \quad \alpha_{0}+\alpha_{1}>0, \quad \beta_{0}+\beta_{1}>0, \quad \alpha_{0}+\beta_{0}>0,
$$

and where $\mathrm{K}, \mathrm{q}, \mathrm{f}$ are given (smooth) functions, with $\mathrm{K}(x)>0, \mathrm{q}(x) \geq 0$ everywhere on $[a, b]$. (Actually, the analysis carries over to more general q, but to avoid nonessential details we will restrict our attention to the simpler case $q \geq 0$.) Under these conditions, it is well known that problem (1.1) admits a unique solution u, whose values are typically obtained only through some sort of discrete approximations such as those provided by finite difference or finite element schemes.

For the discretization of (1.1), we set up an arbitrary grid on the interval $[a, b]$, picking $N+1$ points $a=x_{0}<x_{1}<\cdots<x_{N-1}<x_{N}=b$, called nodes, which divide $[a, b]$ into $N$ subintervals $\left[x_{i-1}, x_{i}\right]$, or cells, with lengths $L_{i-1 / 2}=x_{i}-x_{i-1}$,

\footnotetext{
${ }^{1}$ The authors would like to thank CAPES, CNPq and FAPERGS for their partial support.

${ }^{2}$ greice.lorenzzetti@ufrgs.br, jzingano@mat.ufrgs.br, pzingano@mat.ufrgs.br
} 
whose centers will be denoted by $x_{i-1 / 2}, 1 \leq i \leq N$. (Here, we follow notation in [1], [5].) Throughout the text, it will be convenient to set $x_{-1 / 2} \equiv x_{0}, L_{-1 / 2} \equiv 0$, $x_{N+1 / 2} \equiv x_{N}, L_{N+1 / 2} \equiv 0$, and associate some local length with the nodes, given by $h_{i}:=x_{i+1 / 2}-x_{i-1 / 2}$, i.e.,

$$
h_{i}:=\frac{L_{i-1 / 2}+L_{i+1 / 2}}{2}, \quad 0 \leq i \leq N \quad\left(L_{-1 / 2} \equiv 0, L_{N+1 / 2} \equiv 0\right) .
$$

The corresponding numerical schemes to be considered are then cast in the form

$$
\begin{gathered}
-\mathcal{D}\left(K \cdot \mathcal{G} v^{h}\right)+q \cdot v^{h}=f, \\
\alpha_{0} v_{0}-\alpha_{1} K_{0} \cdot\left(\mathcal{G} v^{h}\right)_{0}=\Gamma_{a}, \quad \beta_{0} v_{N}+\beta_{1} K_{N} \cdot\left(\mathcal{G} v^{h}\right)_{N}=\Gamma_{b},
\end{gathered}
$$

for appropriate difference operators $\mathcal{D}$ ("discrete divergent"), $\mathcal{G}$ ("discrete gradient") and discrete functions $K, q, f$ (or, in more pedantic notation, $K^{h}, q^{h}, f^{h}$, with superscript $h$ referring to the grid) that represent ("project") $\mathrm{K}, \mathrm{q}, \mathrm{f}$ on appropriate grid points; solving (1.3) for $v^{h}$ gives the approximation sought for the exact values $u^{h}$. For example, the standard finite-difference formula ${ }^{3}$

$-\frac{K_{i-1 / 2}}{L_{i-1 / 2}} v_{i-1}+\left(\frac{K_{i-1 / 2}}{L_{i-1 / 2}}+\frac{K_{i+1 / 2}}{L_{i+1 / 2}}+q_{i} h_{i}\right) v_{i}-\frac{K_{i+1 / 2}}{L_{i+1 / 2}} v_{i+1}=h_{i} f_{i}$,

with $1 \leq i \leq N-1$, plus the boundary terms corresponding to (1.1b) above, has $\mathcal{D}, \mathcal{G}$ given by

$$
(\mathcal{D} w)_{i}=\frac{w_{i+1 / 2}-w_{i-1 / 2}}{h_{i}}, \quad(\mathcal{G} z)_{i-1 / 2}=\frac{z_{i}-z_{i-1}}{L_{i-1 / 2}},
$$

while the so-called mimetic method [2], [5]

$-\frac{K_{i-1}}{h_{i-1}} v_{i-3 / 2}+\left(\frac{K_{i-1}}{h_{i-1}}+\frac{K_{i}}{h_{i}}+q_{i-1 / 2} L_{i-1 / 2}\right) v_{i-1 / 2}-\frac{K_{i}}{h_{i}} v_{i+1 / 2}=L_{i-1 / 2} f_{i-1 / 2}$,

with $1 \leq i \leq N$, corresponds to ${ }^{4}$

$$
(\mathcal{D} w)_{i-1 / 2}=\frac{w_{i}-w_{i-1}}{L_{i-1 / 2}}, \quad(\mathcal{G} z)_{i}=\frac{z_{i+1 / 2}-z_{i-1 / 2}}{h_{i}} .
$$

Of foremost importance here are the errors $e^{h}:=v^{h}-u^{h}$ ("solution error"), $E^{h}:=\mathcal{G} v^{h}-\left(\mathbf{u}^{\prime}\right)^{h}$ ("gradient error"), which are related to $\tau^{h}$ ("truncation error") defined by

$$
-\mathcal{D}\left(K \cdot \mathcal{G} u^{h}\right)+q \cdot u^{h}=f+\tau^{h}
$$

at the internal nodes or cell centers, according to the particular scheme, and

$$
\alpha_{0} u_{0}-\alpha_{1} K_{0} \cdot\left(\mathcal{G} u^{h}\right)_{0}=\Gamma_{a}+\tau_{0}, \quad \beta_{0} u_{N}+\beta_{1} K_{N} \cdot\left(\mathcal{G} u^{h}\right)_{N}=\Gamma_{b}+\tau_{N}(1.6 \mathrm{~b})
$$

\footnotetext{
${ }^{3}$ Given $\mathrm{w}:[a, b] \rightarrow \mathbb{R}$, we denote $w_{i}:=\mathrm{w}\left(x_{i}\right), w_{i-1 / 2}:=\mathrm{w}\left(x_{i-1 / 2}\right), w_{i}^{\prime}:=\mathrm{w}^{\prime}\left(x_{i}\right), w_{i+1 / 2}^{\prime \prime}:=$ $\mathrm{w}^{\prime \prime}\left(x_{i+1 / 2}\right)$, etc. Cell averaged values (as in finite element schemes) can also be used. Grid function values at particular grid points are denoted in this work without superscript $h\left(v_{i}, w_{i-1 / 2}\right.$, etc.).

${ }^{4}$ Although (1.4) and (1.5) look similar, they may behave quite differently (see below and [4], [5]).
} 
at the endpoints $x_{0}=a, x_{N}=b$. The relevance of $\tau^{h}$ can be seen from the equations

$$
\begin{gathered}
-\mathcal{D}\left(K \cdot \mathcal{G} e^{h}\right)+q \cdot e^{h}=-\tau^{h} \\
\alpha_{0} e_{0}-\alpha_{1} K_{0} \cdot\left(\mathcal{G} e^{h}\right)_{0}=-\tau_{0}, \quad \beta_{0} e_{N}+\beta_{1} K_{N} \cdot\left(\mathcal{G} e^{h}\right)_{N}=-\tau_{N}
\end{gathered}
$$

relating $\tau^{h}$ to $e^{h}$. In particular, it is a fundamental result that, as the grid is infinitely refined (" $h \rightarrow 0$ "), condition $\tau^{h} \rightarrow 0$ ("consistency") is sufficient to assure $e^{h} \rightarrow 0$ ("convergence") ${ }^{5}$, although it is by no means necessary [2], [4], [7], [8]. One example is given by scheme (1.5), for which ${ }^{6}$

$$
\begin{gathered}
\tau_{i-1 / 2}=K_{i-1 / 2} u_{i-1 / 2}^{\prime \prime}\left(1-\frac{h_{i}+h_{i-1}}{2 L_{i-1 / 2}}\right)-\frac{1}{6} K_{i-1 / 2} u_{i-1 / 2}^{\prime \prime \prime} \frac{h_{i}^{2}-h_{i-1}^{2}}{L_{i-1 / 2}} \\
-\frac{1}{4} K_{i-1 / 2}^{\prime} u_{i-1 / 2}^{\prime \prime}\left(h_{i}-h_{i-1}\right)+O\left(L_{i-3 / 2}^{2}\right)+O\left(L_{i-1 / 2}^{2}\right)+O\left(L_{i+1 / 2}^{2}\right)
\end{gathered}
$$

for $1 \leq i \leq N$ : not only $\tau^{h}$ does not decrease uniformly as $h \rightarrow 0$, it may well grow unboundedly! In spite of such odd behavior, (1.5) turns out to have some nice convergence properties and even a few advantages over the more standard, consistent formula (1.4) [2], [5], [8]. However, it is in general extremely difficult to investigate the behavior of convergent inconsistent schemes by traditional numerical analysis, particularly on rough grids ${ }^{7}[4]$. One nice feature of our approach is that it can handle many such problems almost as easily as it does in case of problems that are simple enough to be satisfactorily tackled by classical analysis. In the next sections, this will be illustrated by applying our procedure to the derivation of sharp error estimates for the mimetic scheme (1.5). The results are easy to describe: one obtains $^{6}$

$$
\begin{gathered}
e_{i-1 / 2}=-\frac{1}{8} \mathbf{u}^{\prime \prime}\left(x_{i-1 / 2}\right) L_{i-1 / 2}^{2}+O\left(\hbar^{2}\right), \quad 1 \leq i \leq N \\
e_{0}=O\left(\hbar^{2}\right), \quad e_{N}=O\left(\hbar^{2}\right) \\
E_{i}=O\left(\hbar^{2}\right), \quad 0 \leq i \leq N
\end{gathered}
$$

with all $O\left(\hbar^{2}\right)$ terms having size bounded by $C \hbar^{2}$ for some positive constant $C$ that depends on the solution $\mathrm{u}$ but is independent of $i$ or the particular grid considered, where $\hbar$ is the grid spacing parameter given by

$$
\hbar=\sqrt{\sum_{j=1}^{N} L_{j-1 / 2}^{3}} .
$$

This is precisely the exact behavior observed in numerical experiments [1], [5], [8].

\footnotetext{
${ }^{5}$ One must note that, in our present setting, consistency is also sufficient for zero-stability [8].

${ }^{6}$ Expressions (1.8), (1.9) are valid provided that $\mathrm{K} \in C^{3}([a, b]), \mathrm{u} \in C^{4}([a, b])$. If $\mathrm{K}$, $\mathrm{u}$ are less smooth, then these must be changed accordingly.

${ }^{7}$ Similar comments apply to the investigation of supraconvergence phenomena in general [3], [7].
} 


\section{Error Analysis for $q=0$}

Our analysis of discrete schemes (1.3) begins with the fundamental case $q=0$. To shorten our exposition, let us discuss the Dirichlet problem only, the other boundary problems being treated in a completely similar way. The starting point in the analysis is to estimate the quantity $\mathcal{G} e^{h}$. Because

$$
K_{i}\left(\mathcal{G} e^{h}\right)_{i}=K_{N}\left(\mathcal{G} e^{h}\right)_{N}-\sum_{j=i+1}^{N} L_{j-1 / 2}\left(\mathcal{D}\left(K \cdot \mathcal{G} e^{h}\right)\right)_{j-1 / 2}
$$

for all $0 \leq i \leq N$, we obtain, using (1.7) and recalling that $\mathrm{q}=0$,

$$
K_{i}\left(\mathcal{G} e^{h}\right)_{i}=K_{N}\left(\mathcal{G} e^{h}\right)_{N}-\sum_{j=i+1}^{N} L_{j-1 / 2} \tau_{j-1 / 2}, \quad 0 \leq i \leq N .
$$

Now, from (1.8), we get

$$
\begin{aligned}
& \sum_{j=i+1}^{N} L_{j-1 / 2} \tau_{j-1 / 2}=O\left(\hbar^{2}\right)+\sum_{j=i+1}^{N} K_{j-1 / 2} u_{j-1 / 2}^{\prime \prime}\left(L_{j-1 / 2}-\frac{h_{j-1}+h_{j}}{2}\right) \\
& -\frac{1}{4} \sum_{j=i+1}^{N} K_{j-1 / 2}^{\prime} u_{j-1 / 2}^{\prime \prime} L_{j-1 / 2}\left(h_{j}-h_{j-1}\right)-\frac{1}{6} \sum_{j=i+1}^{N} K_{j-1 / 2} u_{j-1 / 2}^{\prime \prime \prime}\left(h_{j}^{2}-h_{j-1}^{2}\right)
\end{aligned}
$$

and we proceed by estimating the sums on the right hand side of (2.3): for the first one, setting $\mathbf{w}(x)=\mathrm{K}(x) \mathbf{u}^{\prime \prime}(x)$, we have

$$
\begin{aligned}
\sum_{j=i+1}^{N} K_{j-1 / 2} & u_{j-1 / 2}^{\prime \prime}\left(L_{j-1 / 2}-\frac{h_{j-1}+h_{j}}{2}\right)= \\
= & -\frac{1}{4} \sum_{j=i+1}^{N} w_{j-1 / 2}\left\{\left(L_{j+1 / 2}-L_{j-1 / 2}\right)-\left(L_{j-1 / 2}-L_{j-3 / 2}\right)\right\} \\
= & -\frac{1}{4} \sum_{j=i+1}^{N}\left\{w_{j}\left(L_{j+1 / 2}-L_{j-1 / 2}\right)-w_{j-1}\left(L_{j-1 / 2}-L_{j-3 / 2}\right)\right\} \\
& +\frac{1}{4} \sum_{j=i+1}^{N}\left\{\left(w_{j}-w_{j-1 / 2}\right)\left(L_{j+1 / 2}-L_{j-1 / 2}\right)\right. \\
& +\frac{1}{4} \sum_{j=i+1}^{N}\left(w_{j-1 / 2}-w_{j-3 / 2}\right)\left(L_{j-1 / 2}-L_{j-3 / 2}\right) \\
= & \frac{1}{4} w_{N-1 / 2} L_{N-1 / 2}+\frac{1}{4} w_{i-1 / 2}\left(L_{i+1 / 2}-L_{i-1 / 2}\right) \\
& +\frac{1}{8} \sum_{j=i+1}^{N} w_{j-1}^{\prime}\left(L_{j-1 / 2}^{2}-L_{j-3 / 2}^{2}\right)+O\left(\hbar^{2}\right) \\
= & \frac{1}{4} w_{N-1 / 2} L_{N-1 / 2}+\frac{1}{8} w_{N-1 / 2}^{\prime} L_{N-1 / 2}^{2}+\frac{1}{4} w_{i-1 / 2}\left(L_{i+1 / 2}-L_{i-1 / 2}\right)
\end{aligned}
$$




$$
\begin{gathered}
-\frac{1}{8} w_{i}^{\prime} L_{i-1 / 2}^{2}+O\left(\hbar^{2}\right) \\
=\frac{1}{4} w_{N} L_{N-1 / 2}+\frac{1}{4} w_{i}\left(L_{i+1 / 2}-L_{i-1 / 2}\right)-\frac{1}{8} w_{i}^{\prime} L_{i-1 / 2} L_{i+1 / 2}+O\left(\hbar^{2}\right),
\end{gathered}
$$

so that we obtain

$$
\begin{aligned}
\sum_{j=i+1}^{N} K_{j-1 / 2} & u_{j-1 / 2}^{\prime \prime}\left(L_{j-1 / 2}-\frac{h_{j-1}+h_{j}}{2}\right)= \\
& =\frac{1}{4} K_{N} u_{N}^{\prime \prime} L_{N-1 / 2}+\frac{1}{4} K_{i} u_{i}^{\prime \prime}\left(L_{i+1 / 2}-L_{i-1 / 2}\right)+ \\
& -\frac{1}{8} K_{i}^{\prime} u_{i}^{\prime \prime} L_{i-1 / 2} L_{i+1 / 2}-\frac{1}{8} K_{i} u_{i}^{\prime \prime \prime} L_{i-1 / 2} L_{i+1 / 2}+O\left(\hbar^{2}\right)
\end{aligned}
$$

In a similar way, for the second term in (2.3), setting $\tilde{\mathbf{w}}(x):=\mathrm{K}^{\prime}(x) \mathbf{u}^{\prime \prime}(x)$, we get

$$
\begin{aligned}
& \sum_{j=i+1}^{N} K_{j-1 / 2}^{\prime} u_{j-1 / 2}^{\prime \prime} L_{j-1 / 2}\left(h_{j}-h_{j-1}\right)= \\
& =-\frac{1}{4} \sum_{j=i+1}^{N}\left\{\tilde{w}_{j} L_{j-1 / 2} h_{j}-\tilde{w}_{j-1} L_{j-1 / 2} h_{j-1}\right\}+O\left(\hbar^{2}\right) \\
& =\tilde{w}_{N} L_{N-1 / 2} h_{N}-\tilde{w}_{i} L_{i-1 / 2} h_{i}-\sum_{j=i+1}^{N} \tilde{w}_{j-1}\left(L_{j-1 / 2}-L_{j-3 / 2}\right) h_{j-1} \\
& \quad+O\left(\hbar^{2}\right) \\
& =\tilde{w}_{N} L_{N-1 / 2} h_{N}-\tilde{w}_{i} L_{i-1 / 2} h_{i}-\frac{1}{2} \sum_{j=i+1}^{N}\left\{\tilde{w}_{j} L_{j-1 / 2}^{2}-\tilde{w}_{j-1} L_{j-3 / 2}^{2}\right\} \\
& \quad+O\left(\hbar^{2}\right) \\
& =-\frac{1}{2} \tilde{w}_{i} L_{i-1 / 2} L_{i+1 / 2}+O\left(\hbar^{2}\right),
\end{aligned}
$$

that is,

$$
\sum_{j=i+1}^{N} K_{j-1 / 2}^{\prime} u_{j-1 / 2}^{\prime \prime} L_{j-1 / 2}\left(h_{j}-h_{j-1}\right)=-\frac{1}{2} K_{i}^{\prime} u_{i}^{\prime \prime} L_{i-1 / 2} L_{i+1 / 2}+O\left(\hbar^{2}\right) .
$$

Finally, for the third term in (2.3), we get

$$
\begin{gathered}
\sum_{j=i+1}^{N} K_{j-1 / 2} u_{j-1 / 2}^{\prime \prime \prime}\left(h_{j}^{2}-h_{j-1}^{2}\right)=\frac{1}{4} K_{N-1 / 2} u_{N-1 / 2}^{\prime \prime \prime} L_{N-1 / 2}^{2}-K_{i} u_{i}^{\prime \prime \prime} h_{i}^{2} \\
+O\left(\hbar^{2}\right),
\end{gathered}
$$

so that we have, from (2.3) and (2.4a), (2.4b), (2.4c),

$$
\sum_{j=i+1}^{N} L_{j-1 / 2} \tau_{j-1 / 2}=\frac{1}{4} K_{N} u_{N}^{\prime \prime} L_{N-1 / 2}-\frac{1}{24} K_{N} u_{N}^{\prime \prime \prime} L_{N-1 / 2}^{2}+
$$




$$
+\frac{1}{4} K_{i} u_{i}^{\prime \prime}\left(L_{i+1 / 2}-L_{i-1 / 2}\right)-\frac{1}{8} K_{i} u_{i}^{\prime \prime \prime} L_{i-1 / 2} L_{i+1 / 2}+\frac{1}{6} K_{i} u_{i}^{\prime \prime \prime} h_{i}^{2}+O\left(\hbar^{2}\right) .
$$

Now, for (2.2) to be useful, there still remains to estimate $\left(\mathcal{G} e^{h}\right)_{N}=-e_{N-1 / 2} / h_{N}$. Solving (1.7a) for $e_{N-1 / 2}$ (using, say, Gaussian elimination), we obtain

$$
e_{N-1 / 2}=-\frac{\theta_{N-1}^{[0]} c_{N-1}}{c_{N}+\theta_{N-1}^{[0]} c_{N-1}} \sum_{j=1}^{N} \frac{1}{\theta_{j-1}^{[0]} c_{j-1}} L_{j-1 / 2} \tau_{j-1 / 2}
$$

where $c_{i}=K_{i} / h_{i}$ and

$$
\theta_{i}^{[0]}=\frac{h_{i}}{K_{i}}\left(\sum_{\ell=0}^{i} \frac{h_{\ell}}{K_{\ell}}\right)^{-1}, \quad 0 \leq i \leq N .
$$

The sum on the right hand side of (2.6) can be computed as in (2.3)-(2.5) above: setting

$$
I_{N}:=\sum_{j=0}^{N} \frac{h_{j}}{K_{j}}=\int_{a}^{b} \frac{1}{\mathrm{~K}(x)} d x+O\left(\hbar^{2}\right),
$$

we get

$$
\begin{aligned}
& \sum_{j=1}^{N} \frac{1}{\theta_{j-1}^{[0]} c_{j-1}} K_{j-1 / 2} u_{j-1 / 2}^{\prime \prime}\left(L_{j-1 / 2}-\frac{h_{j-1}+h_{j}}{2}\right)= \\
& =\frac{1}{4} I_{N} K_{N} u_{N}^{\prime \prime} L_{N-1 / 2}+O\left(\hbar^{2}\right), \\
& \sum_{j=1}^{N} \frac{1}{\theta_{j-1}^{[0]} c_{j-1}} K_{j-1 / 2}^{\prime} u_{j-1 / 2}^{\prime \prime} L_{j-1 / 2}\left(h_{j}-h_{j-1}\right)=O\left(\hbar^{2}\right), \\
& \sum_{j=1}^{N} \frac{1}{\theta_{j-1}^{[0]} c_{j-1}} K_{j-1 / 2} u_{j-1 / 2}^{\prime \prime \prime}\left(h_{j}^{2}-h_{j-1}^{2}\right)=\frac{1}{4} I_{N} K_{N} u_{N}^{\prime \prime \prime} L_{N-1 / 2}^{2}+O\left(\hbar^{2}\right),
\end{aligned}
$$

so that we have

$$
\begin{aligned}
\sum_{j=1}^{N} \frac{1}{\theta_{j-1}^{[0]} c_{j-1}} L_{j-1 / 2} \tau_{j-1 / 2}= & \frac{1}{4} I_{N} K_{N} u_{N}^{\prime \prime} L_{N-1 / 2}+ \\
& -\frac{1}{24} I_{N} K_{N} u_{N}^{\prime \prime \prime} L_{N-1 / 2}^{2}+O\left(\hbar^{2}\right) .
\end{aligned}
$$

Also, from (2.7) and (2.8),

$$
\frac{\theta_{N-1}^{[0]} c_{N-1}}{c_{N}+\theta_{N-1}^{[0]} c_{N-1}}=\frac{1}{1+c_{N} \sum_{\ell=0}^{N-1} \frac{h_{\ell}}{K_{\ell}}}=\frac{h_{N}}{K_{N} \sum_{\ell=0}^{N} \frac{h_{\ell}}{K_{\ell}}}=\frac{h_{N}}{I_{N} K_{N}},
$$

which immediately yields, from (2.6) above,

$$
e_{N-1 / 2}=h_{N}\left\{-\frac{1}{4} u_{N}^{\prime \prime} L_{N-1 / 2}+\frac{1}{24} u_{N}^{\prime \prime \prime} L_{N-1 / 2}^{2}+O\left(\hbar^{2}\right)\right\} .
$$


Therefore, we obtain, for $\left(\mathcal{G} e^{h}\right)_{N}$,

$$
\left(\mathcal{G} e^{h}\right)_{N}=\frac{1}{4} u_{N}^{\prime \prime} L_{N-1 / 2}-\frac{1}{24} u_{N}^{\prime \prime \prime} L_{N-1 / 2}^{2}+O\left(\hbar^{2}\right)
$$

so that we get, from (2.1), (2.5) and (2.12), the fundamental estimate

$$
\begin{gathered}
\left(\mathcal{G} e^{h}\right)_{i}=-\frac{1}{4} u_{i}^{\prime \prime}\left(L_{i+1 / 2}-L_{i-1 / 2}\right)-\frac{1}{24} u_{i}^{\prime \prime \prime}\left(L_{i-1 / 2}^{2}-L_{i-1 / 2} L_{i+1 / 2}\right. \\
\left.+L_{i+1 / 2}^{2}\right)+O\left(\hbar^{2}\right)
\end{gathered}
$$

for all $0 \leq i \leq N$. In particular, because $E^{h}$ satisfies

$$
\begin{aligned}
E_{i} & =\left(\mathcal{G} e^{h}\right)_{i}+\left(\mathcal{G} u^{h}\right)_{i}-u_{i}^{\prime} \\
& =\left(\mathcal{G} e^{h}\right)_{i}+\frac{1}{4} u_{i}^{\prime \prime}\left(L_{i+1 / 2}-L_{i-1 / 2}\right)+\frac{1}{48} u_{i}^{\prime \prime \prime} \frac{L_{i-1 / 2}^{3}+L_{i+1 / 2}^{3}}{h_{i}}+O\left(\hbar^{2}\right),
\end{aligned}
$$

we obtain

$$
E_{i}=O\left(\hbar^{2}\right), \quad 0 \leq i \leq N
$$

as claimed. Finally, from the trapezoidal quadrature rule, we have

$$
e_{i-1 / 2}=-\frac{1}{8} u^{\prime \prime}\left(x_{i-1 / 2}\right) L_{i-1 / 2}^{2}+O\left(L_{1 / 2}^{3}+\ldots+L_{i-1 / 2}^{3}\right)+\sum_{j=0}^{i-1} h_{j} E_{j}
$$

for all $1 \leq i \leq N+1$. so that (2.14) gives

$$
e_{i-1 / 2}=-\frac{1}{8} u_{i-1 / 2}^{\prime \prime} L_{i-1 / 2}^{2}+O\left(\hbar^{2}\right), \quad 1 \leq i \leq N
$$

\section{Error Analysis for $q \geq 0$}

We now derive similar estimates for nonnegative $\mathrm{q} \in C^{0}([a, b])$, and same smoothness assumptions on $\mathrm{K}$, u. Given such $\mathrm{q}$, the corresponding solution error will hereafter be denoted by $e^{[\mathrm{q}]}$, to distinguish from the case $\mathbf{q}=0$. Writing $(1.7 \mathrm{a})$ as

$$
A_{h}(\mathbf{q}) e^{[\mathbf{q}]}=-\mathcal{L}_{h} \tau^{h}, \quad A_{h}(\mathbf{q})=A_{h}(0)+Q_{h}(\mathbf{q})
$$

where $\mathcal{L}_{h}=\operatorname{diag}\left(L_{1 / 2}, \ldots, L_{N-1 / 2}\right), \quad Q_{h}(\mathbf{q})=\operatorname{diag}\left(q_{1 / 2} L_{1 / 2}, \ldots, q_{N-1 / 2} L_{N-1 / 2}\right)$, and recalling that $A_{h}(0) e^{[0]}=-\mathcal{L}_{h} \tau^{h}$, we obtain, for the difference $e^{[\mathrm{q}]}-e^{[0]}$, the linear algebraic equation

$$
A_{h}(\mathbf{q})\left(e^{[\mathbf{q}]}-e^{[0]}\right)=-Q_{h} e^{[0]}
$$

Reducing (3.2) to triangular form, we obtain $\hat{A}_{h}(\mathbf{q})\left(e^{[\mathbf{q}]}-e^{[0]}\right)=\hat{\zeta}$, where $\hat{\zeta}$ is given by 


$$
\hat{\zeta}_{i-1 / 2}=-\sum_{j=1}^{i} \frac{\theta_{i-1}^{[\mathrm{q}]} c_{i-1}}{\theta_{j-1}^{[\mathrm{q}]} c_{j-1}} \nu_{i j}^{[\mathrm{q}]} L_{j-1 / 2} q_{j-1 / 2} e_{j-1 / 2}^{[0]}, \quad 1 \leq i \leq N,
$$

with $\left|\nu_{i j}^{[\mathrm{q}]}\right| \leq 1$ for all $i, j$, and positive $\theta_{1}^{[\mathrm{q}]}, \theta_{2}^{[\mathrm{q}]}, \ldots$ given recursively by $\theta_{0}^{[\mathrm{q}]}=1$ and

$$
\theta_{i}^{[\mathrm{q}]}=\frac{c_{i-1} \theta_{i-1}^{[\mathrm{q}]}+q_{i-1 / 2} L_{i-1 / 2}}{c_{i-1} \theta_{i-1}^{[\mathrm{q}]}+c_{i}+q_{i-1 / 2} L_{i-1 / 2}}, \quad 1 \leq i \leq N-1 .
$$

Thus, we have

that is,

$$
\begin{aligned}
\left|\hat{\zeta}_{i-1 / 2}\right| & \leq \theta_{i-1}^{[\mathrm{q}]} c_{i-1} \sum_{j=1}^{i} \frac{1}{\theta_{j-1}^{[\mathrm{q}]} c_{j-1}} L_{j-1 / 2}\|\mathrm{q}\|_{\text {sup }}\left|e_{j-1 / 2}^{[0]}\right| \\
& \leq \theta_{i-1}^{[\mathrm{q}]} c_{i-1}\|\mathrm{q}\|_{\sup } \sum_{\ell=1}^{i} \frac{h_{\ell}}{K_{\ell}} \sum_{j=1}^{i} L_{j-1 / 2}\left|e_{j-1 / 2}^{[0]}\right|, \quad 1 \leq i \leq N,
\end{aligned}
$$

$$
\left|\hat{\zeta}_{i-1 / 2}\right| \leq \theta_{i-1}^{[\mathrm{q}]} c_{i-1} I_{N}\|\mathrm{q}\|_{\sup } \sum_{j=1}^{N} L_{j-1 / 2}\left|e_{j-1 / 2}^{[0]}\right|, \quad 1 \leq i \leq N,
$$

where $\|\mathrm{q}\|_{\text {sup }}$ denotes the supnorm of $\mathbf{q}$ on $[a, b]$. This immediately gives

$$
\left\|e^{[\mathrm{q}]}-e^{[0]}\right\|_{\sup } \leq I_{N}\|\mathrm{q}\|_{\sup } \sum_{j=1}^{N} L_{j-1 / 2}\left|e_{j-1 / 2}^{[0]}\right|, \quad 1 \leq i \leq N,
$$

so that $\left\|e^{[\mathrm{q}]}-e^{[0]}\right\|_{\text {sup }}=O\left(\hbar^{2}\right)$ by $(2.15)$. Therefore,

$$
e_{i-1 / 2}^{[\mathrm{q}]}=-\frac{1}{8} \mathbf{u}^{\prime \prime}\left(x_{i-1 / 2}\right) L_{i-1 / 2}^{2}+O\left(\hbar^{2}\right), \quad 1 \leq i \leq N
$$

where the size of the constant in the term $O\left(\hbar^{2}\right)$ depends on the values of $\mathrm{q}, \mathrm{K}$, $1 / \mathrm{K}$ and of derivatives $\mathrm{K}^{(\ell)}, \mathrm{u}^{(1+\ell)}, 0 \leq \ell \leq 3$, on $[a, b]$.

It still remains to estimate $E^{[\mathrm{q}]}, \mathcal{G} e^{[\mathrm{q}]}$, and, now that (3.6) has been obtained, this can be done as in Section 2: from (2.1), we have, for $0 \leq i \leq N$,

$$
K_{i}\left(\mathcal{G} e^{[\mathrm{q}]}\right)_{i}=K_{N}\left(\mathcal{G} e^{[\mathrm{q}]}\right)_{N}-\sum_{j=i+1}^{N} L_{j-1 / 2} q_{j-1 / 2} e_{j-1 / 2}^{[\mathrm{q}]}-\sum_{j=i+1}^{N} L_{j-1 / 2} \tau_{j-1 / 2},
$$

where the first sum has size $O\left(\hbar^{2}\right)$, by (3.6), and the last has already been computed, see (2.5) above. As to $K_{N}\left(\mathcal{G} e^{[\mathrm{q}]}\right)_{N}$, we proceed as we did before, computing $e_{N-1 / 2}^{[\mathbf{q}]}$ to third order accuracy. From $(3.1)$, we have $A_{h}(0) e^{[\mathbf{q}]}=b(\mathrm{u})-Q_{h}(\mathbf{q}) e^{[\mathbf{q}]}$, where $b(\mathrm{u}):=-\mathcal{L}_{h} \tau^{h}$; after elimination, this becomes $\hat{A}_{h}(0) e^{[\mathrm{q}]}=\hat{b}(\mathrm{u})-\hat{\zeta}$, with $\hat{\zeta}=\left(\hat{\zeta}_{1 / 2}, \ldots, \hat{\zeta}_{N-1 / 2}\right)$ given by

$$
\hat{\zeta}_{i-1 / 2}=-\sum_{j=1}^{i} \frac{\theta_{i-1}^{[0]} c_{i-1}}{\theta_{j-1}^{[0]} c_{j-1}} L_{j-1 / 2} q_{j-1 / 2} e_{j-1 / 2}^{[\mathrm{q}]}, \quad 1 \leq i \leq N,
$$



where $c_{\ell}:=K_{\ell} / h_{\ell}$. This gives $\left|\hat{\zeta}_{i-1 / 2}\right| \leq \theta_{i-1}^{[0]} c_{i-1} I_{N}\|\mathrm{q}\|_{\text {sup }} \sum_{j=1}^{i} L_{j-1 / 2}\left|e_{j-1 / 2}^{[\mathrm{q}]}\right|$
and therefore, by $(2.10)$,

$$
\begin{gathered}
\left|\left(\hat{A}_{h}(0)^{-1} \hat{\zeta}\right)_{N-1 / 2}\right| \leq \frac{\theta_{N-1}^{[0]} c_{N-1}}{c_{N}+\theta_{N-1}^{[0]} c_{N-1}} I_{N}\|\mathrm{q}\|_{\sup } \sum_{j=1}^{N} L_{j-1 / 2}\left|e_{j-1 / 2}^{[\mathrm{q}]}\right| \\
=\frac{h_{N}}{K_{N}}\|\mathrm{q}\|_{\sup } \sum_{j=1}^{N} L_{j-1 / 2}\left|e_{j-1 / 2}^{[\mathrm{q}]}\right| .
\end{gathered}
$$

For $e^{[0]}=A_{h}(0)^{-1} b(\mathrm{u})=\hat{A}_{h}(0)^{-1} \hat{b}(\mathrm{u})$, we get $e_{N-1 / 2}^{[\mathrm{q}]}=e_{N-1 / 2}^{[0]}-\left(\hat{A}_{h}(0)^{-1} \hat{\zeta}\right)_{N-1 / 2}$, so that, by (2.6), (2.11) and (3.6), we obtain

$$
e_{N-1 / 2}^{[\mathrm{q}]}=h_{N}\left\{-\frac{1}{4} u_{N}^{\prime \prime} L_{N-1 / 2}+\frac{1}{24} u_{N}^{\prime \prime \prime} L_{N-1 / 2}^{2}+O\left(\hbar^{2}\right)\right\} .
$$

This gives

$$
\left(\mathcal{G} e^{[\mathrm{q}]}\right)_{N}=\frac{1}{4} u_{N}^{\prime \prime} L_{N-1 / 2}-\frac{1}{24} u_{N}^{\prime \prime \prime} L_{N-1 / 2}^{2}+O\left(\hbar^{2}\right),
$$

so that we obtain, from (2.5), (3.6), (3.7) and (3.9a),

$$
\begin{gathered}
\left(\mathcal{G} e^{[\mathrm{q}]}\right)_{i}=-\frac{1}{4} u_{i}^{\prime \prime}\left(L_{i+1 / 2}-L_{i-1 / 2}\right)-\frac{1}{24} u_{i}^{\prime \prime \prime}\left(L_{i-1 / 2}^{2}-L_{i-1 / 2} L_{i+1 / 2}\right. \\
\left.+L_{i+1 / 2}^{2}\right)+O\left(\hbar^{2}\right)
\end{gathered}
$$

for all $0 \leq i \leq N$. Since $E^{[\mathrm{q}]}=\mathcal{G} e^{[\mathrm{q}]}+\mathcal{G} u^{h}-u^{\prime}$, we finally obtain

$$
E_{i}^{[\mathbf{q}]}=O\left(\hbar^{2}\right), \quad 0 \leq i \leq N,
$$

and the argument is complete.

Acknowledgements. The authors would like to express their gratitude to Prof. Stanly L. Steinberg (University of New Mexico, Albuquerque, USA) for his interest in this work.

Resumo. Neste trabalho é apresentado um procedimento de análise simples para a investigação detalhada dos erros de aproximação em malhas arbitrárias decorrentes de métodos de diferenças finitas usados na discretização de problemas de SturmLiouville unidimensionais. Como ilustração, um esquema inconsistente (mas convergente) é examinado, com estimativas precisas dos erros de solução e gradiente.

\section{References}

[1] J.M. Hyman, M. Shashkov, S.L. Steinberg, The numerical solution of diffusion problems in strongly heterogeneous non-isotropic materials, J. Comput. Phys., 132 (1997), $130-148$. 
[2] J.M. Hyman, S.L. Steinberg, The convergence of mimetic discretization for rough grids, Comput. Math. Applic., 47 (2004), 1565-1610.

[3] T.A. Manteufell, A.B. White, Jr., The numerical solution of second-order boundary value problems on nonuniform meshes, Math. Comp., 47 (1986), $511-535$.

[4] A.A. Samarskii, "The Theory of Difference Schemes", M. Dekker, New York, 2001.

[5] M. Shashkov, "Conservative Finite-difference Methods on General Grids", CRC Press, Boca Raton, 1996.

[6] S.L. Steinberg, Applications of high-order discretizations to boundary-value problems, Comput. Meth. Appl. Math., 4 (2004), 228-261.

[7] B. Swartz, H.- O. Kreiss, T.A. Manteufell, B. Wendroff, A.B. White Jr., Supraconvergent schemes on irregular grids, Math. Comp., 47 (1986), 537-554.

[8] J.P. Zingano, "Convergence of Mimetic Methods for Sturm-Liouville Problems on General Grids", Ph.D. Thesis, University of New Mexico, Albuquerque, NM, 2003. 